\title{
The Prevalence of Nomophobia by Population and by Research Tool: A Systematic Review, Meta-Analysis, and Meta-Regression
}

\author{
Ali Humood ${ }^{1}$ D , Noor Altooq ${ }^{1}$, Abdullah Altamimi ${ }^{1}$, Hasan Almoosawi ${ }^{1}$, Maryam Alzafiri ${ }^{1}$, \\ Nicola Luigi Bragazzi ${ }^{2}$ D, Mariwan Husni ${ }^{1}$ and Haitham Jahrami ${ }^{1,3, * \mathbb{D}}$ \\ 1 College of Medicine and Medical Science, Arabian Gulf University, Manama 329, Bahrain; \\ alihumoodhasan@gmail.com (A.H.); nonatouq-98@live.com (N.A.); aaltamimimd@gmail.com (A.A.); \\ hassan7447@hotmail.com (H.A.); maryamalzafiri@gmail.com (M.A.); mariwanh@agu.edu.bh (M.H.) \\ 2 Laboratory for Industrial and Applied Mathematics (LIAM), Department of Mathematics and Statistics, \\ York University, Toronto, ON M3J 1P3, Canada; robertobragazzi@gmail.com \\ 3 Ministry of Health, Manama 410, Bahrain \\ * Correspondence: hjahrami@health.gov.bh
}

check for updates

Citation: Humood, A.; Altooq, N.; Altamimi, A.; Almoosawi, H.; Alzafiri, M.; Bragazzi, N.L.; Husni, M.; Jahrami, H. The Prevalence of Nomophobia by Population and by Research Tool: A Systematic Review, Meta-Analysis, and Meta-Regression. Psych 2021, 3, 249-258. https:// doi.org/10.3390/psych3020019

Academic Editor: Gergely Fehér

Received: 17 May 2021

Accepted: 18 June 2021

Published: 21 June 2021

Publisher's Note: MDPI stays neutral with regard to jurisdictional claims in published maps and institutional affiliations.

Copyright: (c) 2021 by the authors. Licensee MDPI, Basel, Switzerland. This article is an open access article distributed under the terms and conditions of the Creative Commons Attribution (CC BY) license (https:/ / creativecommons.org/licenses/by/ $4.0 /)$.
Abstract: Background: No systematic review or meta-analysis has yet been performed to examine the global prevalence of nomophobia by population, by instrument. Thus, this review was performed to estimate the prevalence of nomophobia by severity. Methods: American Psychological Association PsycINFO, Cochrane, Cumulative Index to Nursing and Allied Health Literature (CINAHL), EBSCOhost, EMBASE, MEDLINE, ProQuest Medical, ScienceDirect, Scopus, and Web of Science from inception of each respective database to second week of January 2021 were used. There was no language restriction. The random-effect meta-analysis model was used with the DerSimonian and Laird methodology was used for computation. Results: Twenty papers, involving 12,462 participants from ten countries, were evaluated for meta-analysis. The prevalence of moderate to severe nomophobia is $70.76 \%$ [ $95 \%$ CI $62.62 \%$; $77.75 \%$ ]. The prevalence of severe nomophobia is $20.81 \%$ [ $95 \%$ CI $15.45 \% ; 27.43 \%$ ]. University students appeared to be the highest group affected with a prevalence of severe nomophobia $25.46 \%$ [95\% CI 18.49\%; 33.98\%]. Meta-regressions of severe nomophobia showed that age and sex were not a successful predictor of severe nomophobia $\beta=-0.9732, p=0.2672$ and $\beta=-0.9732, p=0.4986$. Conclusions: The prevalence of severe nomophobia is approximately $21 \%$ in the general adult population. University students appeared to be the most impacted by the disorder.

Keywords: anxiety; nomophobia; no-mobile-phobia; NMP-Q

\section{Introduction}

The mobile phone (MP) industry has come a long way in progress and creativity. This has incorporated the use of mobile phones in all aspects of daily living and at almost all levels of human activities, including entertainment, business, acquisition of knowledge, and social networking. These devices are always developing to become more appealing, compelling, and quicker, and mobile firms are vying to give us new models with more memory, better cameras, and batteries, while the number of applications and services available is also growing, making us more reliant on them.

Despite the massive advance and benefits MPs have promoted in the world, they are associated with several physical and psychosocial conditions, such as depression, anxiety, poor attention, and reduced occupational performance [1]. The unlimited and exaggerated use of MPs has caused the emergence of a new psychiatric disorder termed as "Nomophobia", an emerging problem of the modern era [2]. Nomophobia, which refers to "No-mobile-phobia", is described (based on Cambridge Dictionary) as experiencing intense fear anxiety, stress, and discomfort [3], due to the idea of being without a mobile phone or the inability to use it. Nomophobia has its original roots in the United Kingdom [4]. 
The UK post office conducted a study in 2008, which found that around $53 \%$ of people in the United Kingdom suffered irrational fear and anxiety when being disconnected from their MPs [4]. The prevalence of nomophobia in both developed and developing countries ranges between $77 \%$ and $99 \%$ and is most prevalent among the young adult populations [5]. Nomophobia affects $18.5-73 \%$ of college students [2,6]. Individuals with nomophobia share certain typical characteristics, such as the use of multiple mobile phones and chargers, the frequent monitoring of screens to check for notifications, and keeping mobile phones close to them during sleep [7]. People with nomophobia seem to avoid face-to-face interaction and prefer a world of virtual connection $[7,8]$. Various clinical manifestations have been observed in people labeled as having nomophobia. These include tachycardia, sweating, respiratory distress, disorientation, and agitation [6,7]. The Nomophobia Questionnaire (NMP-Q), which consists of 20 items, measures four dimensions of nomophobia, which are "not being able to communicate", "losing connectedness", "not being able to access information", and "giving up convenience" [9]. It has been shown that nomophobia is more prevalent in people with depression, anxiety, low self-confidence, and other comorbid psychiatric illnesses [1]. Although nomophobia is highly prevalent and associated with various health implications, it is still not a qualified phobia and not included as part of the Diagnostic and Statistical Manual of Mental Disorders (DSM-5) [10].

No systematic review and meta-analysis have yet been performed to examine the global pooled prevalence of nomophobia among different populations. To address this knowledge gap, we performed a comprehensive systematic review, meta-analysis, and meta-regression of the prevalence of nomophobia among different populations to explain certain areas of considerable concern in the nomophobia literature. These findings may prove helpful in developing public health strategies for screening and prevention to lower the negative outcome related to nomophobia.

\section{Materials and Methods}

This systematic review and meta-analysis were guided by the preferred reporting items for systematic reviews and metanalyses (PRISMA) statement [11].

\subsection{Data Sources and Searches}

Two members of our review team (H.J and A.H) independently conducted an electronic literature search using five databases; MEDLINE, EMBASE, ScienceDirect, PsychINFO, and ProQuest Medical, and Google Scholar engine.

The following keywords were used: 'nomophobi', 'no-mo-phobia', 'no mobile phobia', 'mobile phone phobia', 'mobile phone addiction', 'mobile phone addiction' and 'prevalence'. The search included all possible search fields (title, abstract, keywords, main text). The search was not limited to any language or any other limits during the search stage, and we searched for papers from the inception of the databases until the second week of January 2021. The final search results were exported into a Microsoft Excel spreadsheet 2019 to be refined and remove duplicates.

The review team manually screened the references of the identified papers for potential inclusion in the review.

\subsection{Study Selection}

Our review included observational studies that aimed to study the prevalence of nomophobia among different populations. To be included in the analyses, research studies had to meet the following criteria: (1) published in the English language, (2) date of publication before the second week of January 2021, (3) investigated the prevalence of nomophobia using a validated screening tool or researcher-developed instrument. Exclusion criteria were: case reports and case series, editorials, and reviews. The flow diagram of study inclusion is shown in Figure 1. 


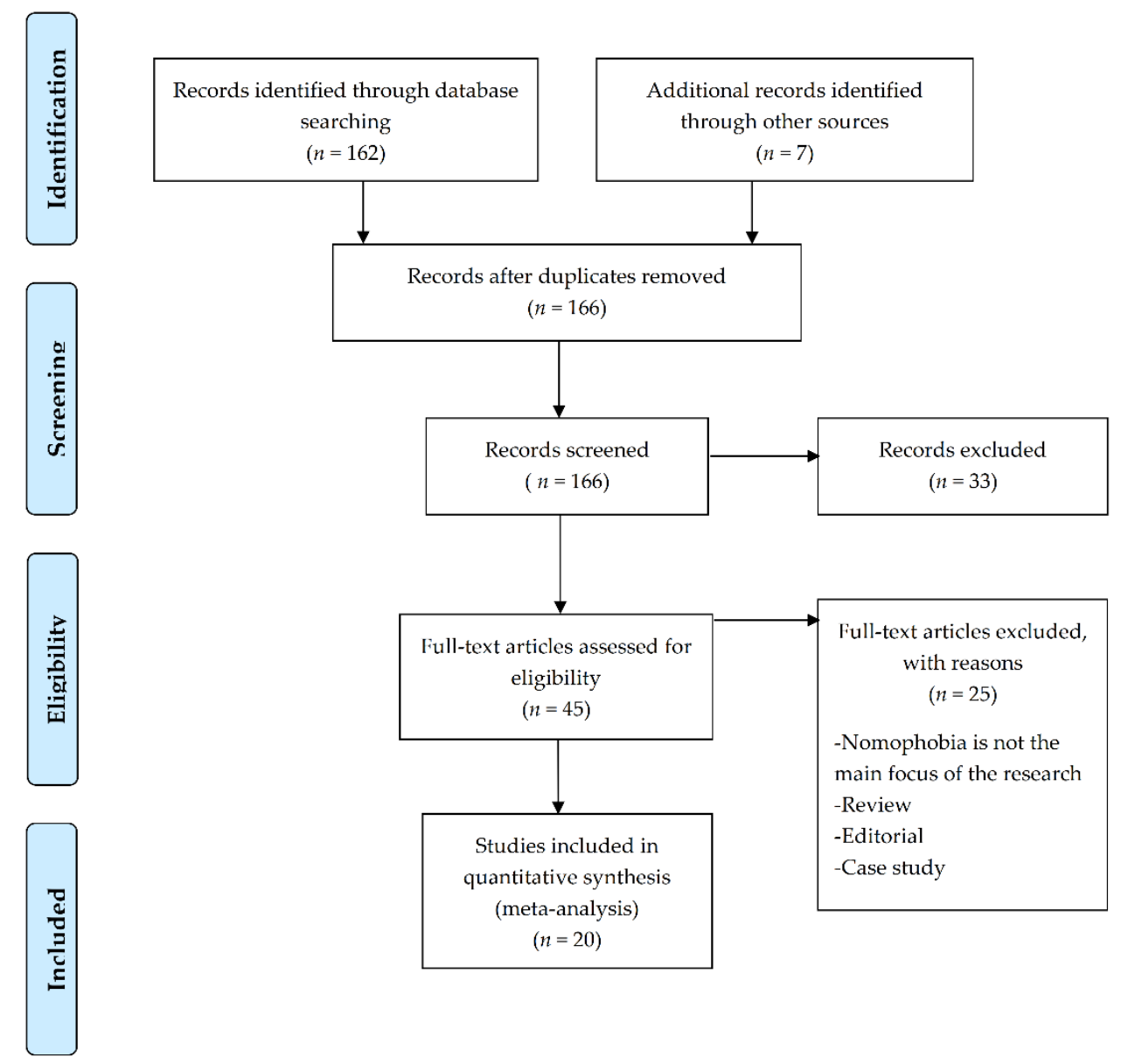

Figure 1. Preferred reporting items for systematic reviews and meta-analyses flow diagram of study inclusion.

\subsection{Outcomes and Measures}

The anticipated outcome of this systematic review and meta-analysis was to report the prevalence of nomophobia in different populations. Five members of the review team (AH, NT, HM, AT, and MZ) independently screened titles, abstracts, and full texts and assessed studies for eligibility criteria, as well as performed the data extraction and research summary. Any disagreements were resolved through discussion with a senior author $(\mathrm{HJ})$ to reach a consensus.

\subsection{Data Extraction}

For each paper, two reviewers independently extracted the data (AH, NT, HM, AT, and MZ). The following information was extracted and tabulated systematically: title, authors, DOI (citation), document type, date of publication, country, sample size, event rate (by severity), mean age, the proportion of male participants, research tool utilized, educational level, occupation, other phobias, comorbid psychiatric problem.

\subsection{Quality Evaluation}

Two reviewers independently assessed the methodological quality of the studies using the Newcastle-Ottawa Scale. Quality assessment was also performed in parallel with data extraction by the same independent two authors that reviewed each study.

\subsection{Data Synthesis and Analysis}

Data were synthesized for the meta-analysis using the using random-effects model according to the DerSimonian-Laird method. We reported the results of the overall prevalence rate and corresponding $95 \%$ confidence intervals. We performed a detailed analysis of 
the heterogeneity using $I^{2}$, Cochran $(Q)$ statistic test, $H$ test, tau, and FMP $\left(\tau^{2}\right)$. A jackknife sensitivity analysis was performed by iteratively removing one study at a time to confirm that our findings were not driven by any single study. Funnel plots are a visual tool for examining publication bias in meta-analysis. A funnel plot is a graph that is widely used in systematic reviews and meta-analyses to assess for the presence of publication bias [12]. It is assumed that, in the absence of publication bias, studies with high accuracy will be plotted around the average, while studies with poor precision will be scattered evenly on both sides of the average, resulting in an approximately funnel-shaped distribution. If the form deviates from this, it might be a sign of publishing bias [12].

Subgroup analysis and meta-regression were performed to examine the influence of different moderators. Subgroup analysis was performed to investigate the different rates of nomophobia by research tool particularly to compare NMP-Q with other research tools. Subgroup analysis was also performed to investigate the different rates of nomophobia by population. The meta-regression technique was used to examine the influence of participants' age and sex on the global prevalence of nomophobia. All data analyses were performed using $\mathrm{R}$ programming language for statistical computing version 4.0.3 [13]. The packages "metafor" [14] was used for all of the analyses and visualizations.

\section{Results}

The present review included a total of 20 empirical research articles; $\mathrm{K}=20$ involving $\mathrm{N}=12,462$ participants were included in the analyses. Studies came from 10 different countries. India contributed with seven studies [2,3,15-20], Iran [21,22], Turkey [1,23], and Pakistan [24,25] contributed each with two studies, and Australia [26], Bahrain [8], Israel [27], Italy [28], Kuwait [29], and USA [30] each contributed with one study. Fourteen studies $[2,15-17,19-25,28-30](70 \%)$ of the studies recruited university students as participants, while four studies $[8,18,27,28](20 \%)$ recruited young adults and only two studies $[1,3]$ $(10 \%)$ recruited high school students. The NMP-Q was used in 12 studies $[1,3,8,17,19,24-30]$ $(60 \%)$, and the remaining used other method to assess the nomophobia, mainly researcherdeveloped research tools. Researcher studies reported the prevalence of nomophobia by severity-mild, moderate, and severe. Selected characteristics, methods, and major findings of the included studies in this review including quality assessment are presented in Table 1.

Random-effects meta-analysis of the prevalence of moderate to severe nomophobia in all populations using all research tools reveals that the prevalence of moderate to severe nomophobia is $70.76 \%$ [ $95 \%$ CI $62.62 \% ; 77.75 \%]$. Quantifying heterogeneity shows that $\tau^{2}=0.6547$ [0.5301; 2.4082]; $\operatorname{tau}=0.8092[0.7280 ; 1.5518], I^{2}=98.7 \%[98.4 \% ; 98.9 \%] ; \mathrm{H}=8.65$ [7.91; 9.46]. Test of heterogeneity: $Q(\mathrm{df}=19) 1421.67, p=0.0001$. Figure 2 shows a Forest plot of the prevalence of moderate to severe nomophobia. Visual inspection to funnel plot revealed no evidence of publication bias. Sensitivity analysis was performed by removing one study at time to investigate if the results were driven by one study. Results from the sensitivity analysis revealed that no one study influence the prevalence rate by more than $2 \%$, suggesting that the studies have robust methodologies and no study should be removed from further analyses.

The prevalence of severe nomophobia in all of the studies $(K=20, N=12462)$ reveals that the prevalence of severe nomophobia is $20.81 \%$ [ $95 \%$ CI $15.45 \% ; 27.43 \%$ ]. Quantifying heterogeneity shows that $\tau^{2}=0.6640$ [0.4600; 1.9278]; tau $=0.8148$ [0.6782; 1.3884], $I^{2}=98.1 \%[97.7 \% ; 98.5 \%] ; \mathrm{H}=7.29[6.60 ; 8.06]$. Test of heterogeneity show that $Q(\mathrm{df}=19)$ $1010.28, p=0.001$. Figure 3 shows the Forest plot of the prevalence of severe nomophobia. Visual inspection to funnel plot revealed no evidence of publication bias, results of the funnel plot is presented in Figure 4.

Subgroup analysis of the prevalence of severe nomophobia by research tools showed that the pooled prevalence of severe nomophobia using NMP-Q was 16.80\% [95\% CI 11.835; $23.30 \%$ ] $\tau^{2}=0.5038$, tau $=0.7098$ while with other research tools, it was $28.36 \%$ [95\% CI $16.16 ; 44.84 \%] \tau^{2}=1.0427$, tau 1.0211. The test for subgroup differences (random-effects 
model) was $Q=2.54 p=0.11$. Figure 5 shows the Forest plot of the prevalence of severe nomophobia by research tool.

Subgroup analysis of the prevalence of severe nomophobia by population showed that the pooled prevalence of severe nomophobia in young adults was $15.15 \%$ [95\% CI $10.25 \%$; $21.83 \%$ ], in university students $25.46 \%$ [ $95 \%$ CI $18.49 \%$; $33.98 \%$ ], and in high school students $8.49 \%$ [ $95 \% 4.19 \% ; 16.47 \%$ ]. The $\tau^{2}$ and tau were: 0.1845 and $0.4295,0.5851$ and 0.7649 , and 0.2863 and 0.5351 , respectively. Figure 6 shows a Forest plot of the prevalence of severe nomophobia by population.

Meta-regressions of severe nomophobia showed that age and sex were not a successful predictor of severe nomophobia $\beta=-0.9732, p=0.2672$ and $\beta=-0.9732, p=0.4986$.

Table 1. Selected characteristics, methods, and major findings of the included studies in this review.

\begin{tabular}{|c|c|c|c|c|c|c|c|c|c|c|}
\hline \multirow{2}{*}{ Study } & \multirow{2}{*}{ Country } & \multirow{2}{*}{ Participants } & \multirow{2}{*}{$\begin{array}{l}\text { Mean } \\
\text { Age }\end{array}$} & \multirow{2}{*}{$\%$ Male } & \multirow{2}{*}{ Tool } & \multirow{2}{*}{$\begin{array}{l}\text { Quality } \\
\text { Score }\end{array}$} & \multirow{2}{*}{ Sample } & \multicolumn{3}{|c|}{ Nomophobia Cases (n) } \\
\hline & & & & & & & & Mild & Moderate & Severe \\
\hline Adawi 2019 [27] & Israel & Young adults & 27.91 & 0.500 & NMP-Q & 6 & 403 & 206 & 167 & 30 \\
\hline Al-Balhan 2018 [29] & Kuwait & University students & 20.00 & 0.500 & NMP-Q & 8 & 512 & 92 & 288 & 132 \\
\hline Batool 2020 [24] & Pakistan & University students & 20.29 & 0.220 & NMP-Q & 6 & 300 & 16 & 204 & 80 \\
\hline Borkotoky 2019 [18] & India & Young adults & 23.00 & 0.480 & Other & 6 & 100 & NR & NR & 25 \\
\hline Bragazzi 2019 [28] & Italy & University students & 27.91 & 0.395 & NMP-Q & 6 & 403 & 206 & 167 & 30 \\
\hline Cain 2019 [30] & USA & University students & 39.00 & 0.354 & NMP-Q & 6 & 192 & 47 & 109 & 35 \\
\hline Daei 2019 [21] & Iran & University students & 23.33 & 0.410 & Other & 6 & 320 & 63 & 235 & 22 \\
\hline Darvishi 2019 [22] & Iran & University students & 23.56 & 0.270 & Other & 6 & 100 & NR & NR & 45 \\
\hline Dasgupta 2017 [19] & India & University students & 21.00 & 0.340 & NMP-Q & 7 & 608 & NR & NR & 265 \\
\hline Dixit 2010 [2] & India & University students & 22.50 & 0.470 & Other & 6 & 200 & NR & 163 & 37 \\
\hline Gurbuz 2020 [23] & Turkey & University students & 22.00 & 0.220 & Other & 6 & 400 & 80 & 286 & 34 \\
\hline Jahrami 2021 [8] & Bahrain & Young adults & 27.20 & 0.460 & NMP-Q & 8 & 654 & 43 & 478 & 133 \\
\hline Jilisha 2020 [17] & India & University students & 19.00 & 0.410 & NMP-Q & 7 & 774 & 161 & 422 & 182 \\
\hline Kaviani 2020 [26] & Australia & Young adults & 40.00 & 0.470 & NMP-Q & 7 & 2838 & 1059 & 1381 & 375 \\
\hline Mallya 2018 [15] & India & University students & 18.00 & 0.386 & Other & 6 & 145 & 11 & 8 & 126 \\
\hline Mengi 2020 [16] & India & University students & 22.00 & 0.758 & Other & 6 & 600 & 163 & 196 & 241 \\
\hline Prasad 2017 [20] & India & University students & 21.99 & 0.471 & Other & 6 & 554 & NR & 234 & 138 \\
\hline Schwaiger 2020 [25] & Pakistan & University students & 23.23 & 0.236 & NMP-Q & 7 & 156 & 16 & 82 & 36 \\
\hline Sharma 2019 [3] & India & High school & 15.50 & 0.670 & NMP-Q & 7 & 1386 & 569 & 303 & 82 \\
\hline Yavuz 2019 [1] & Turkey & High school & 15.42 & 0.470 & NMP-Q & 7 & 1817 & 730 & 856 & 217 \\
\hline
\end{tabular}

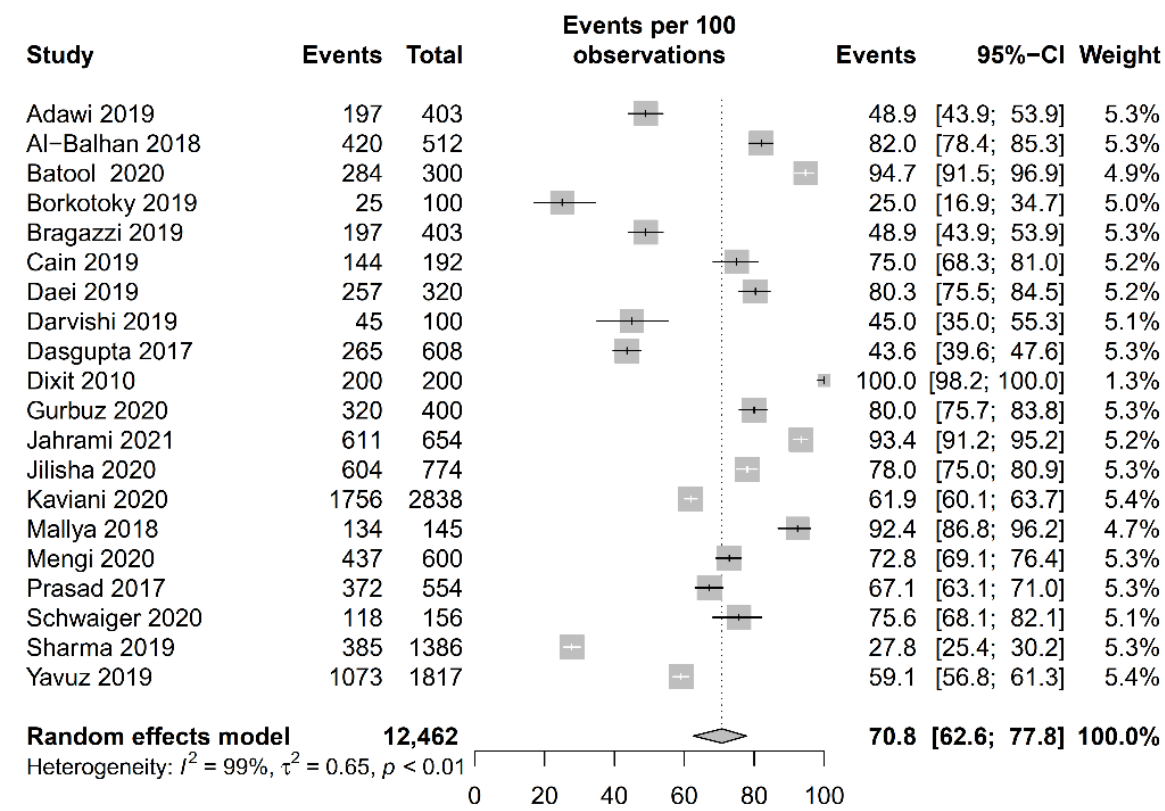

Figure 2. Meta-analysis of the prevalence of moderate-severe nomophobia (all populations, all measures). 


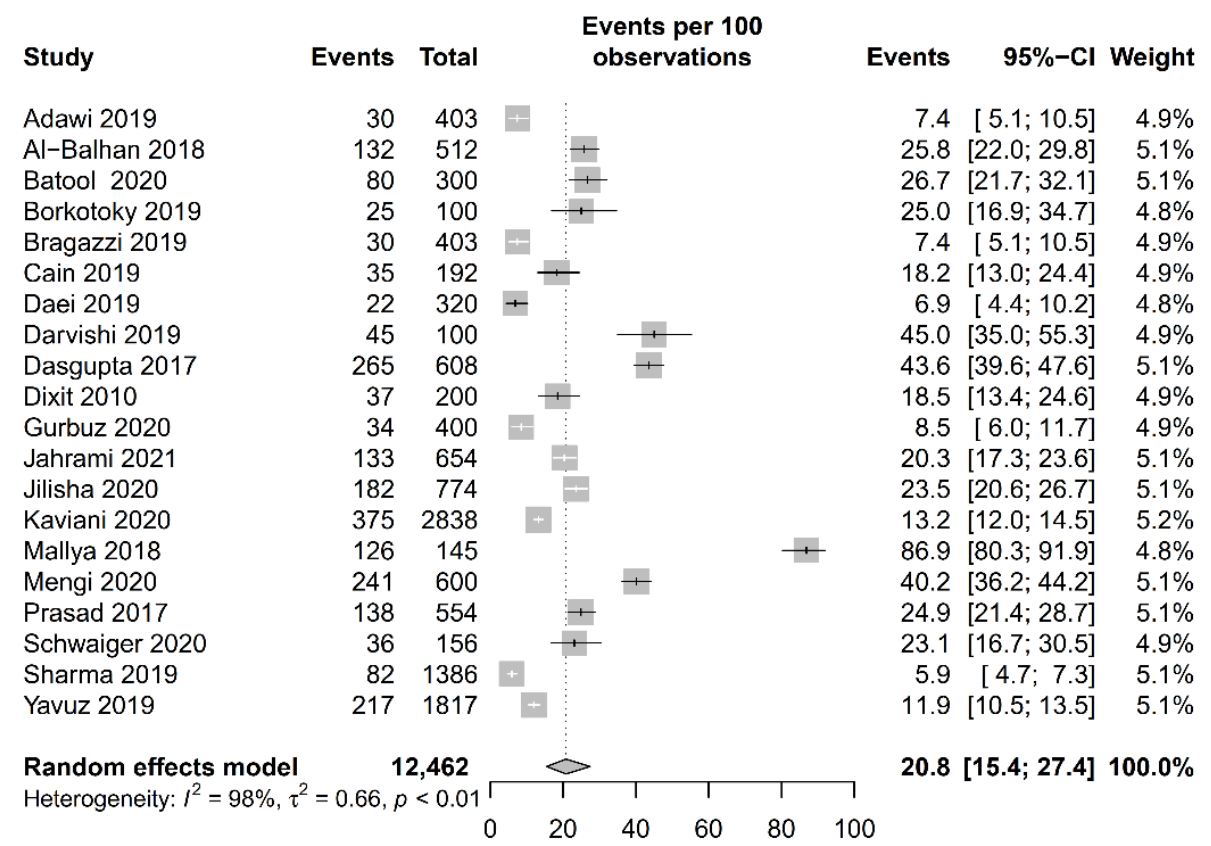

Figure 3. Meta-analysis of the prevalence of severe nomophobia (all populations, all measures).

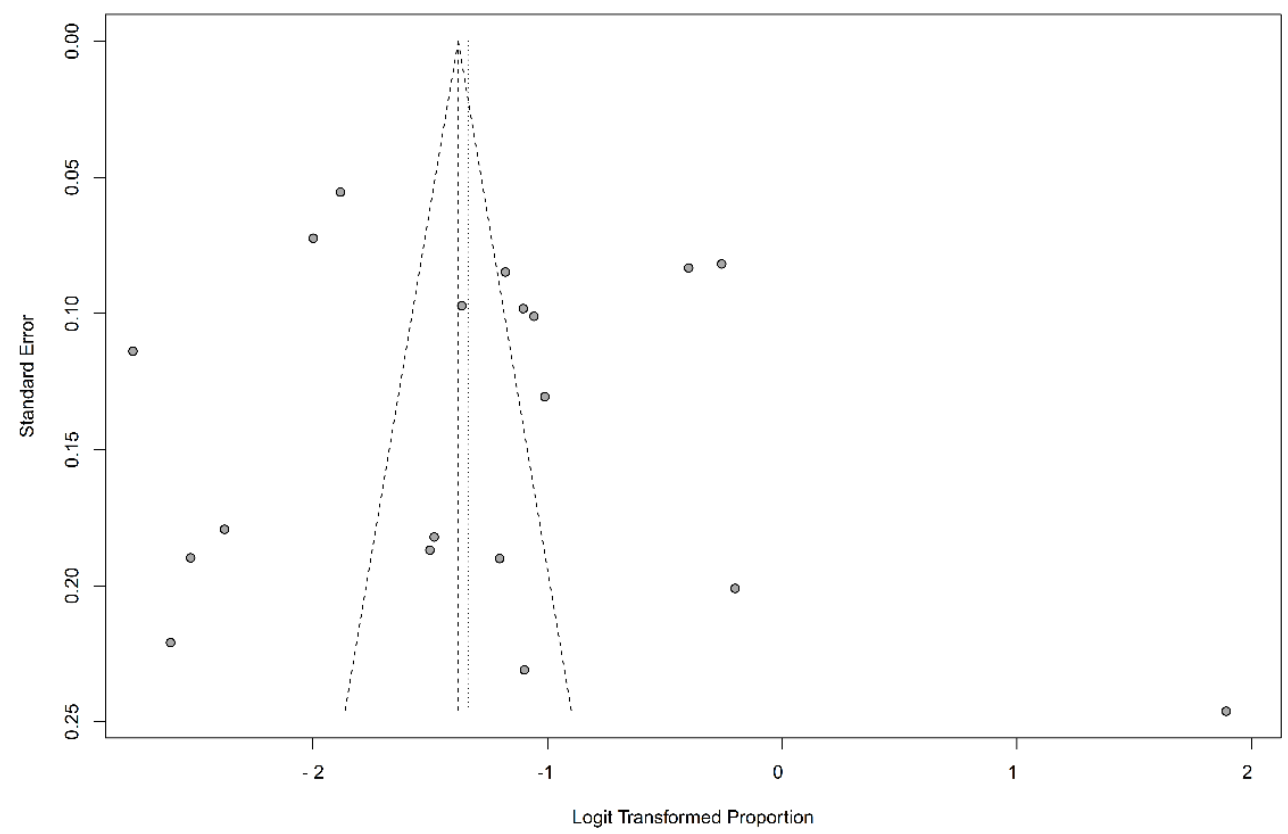

Figure 4. Funnel plot of the prevalence of moderate-severe nomophobia (all populations, all measures). 


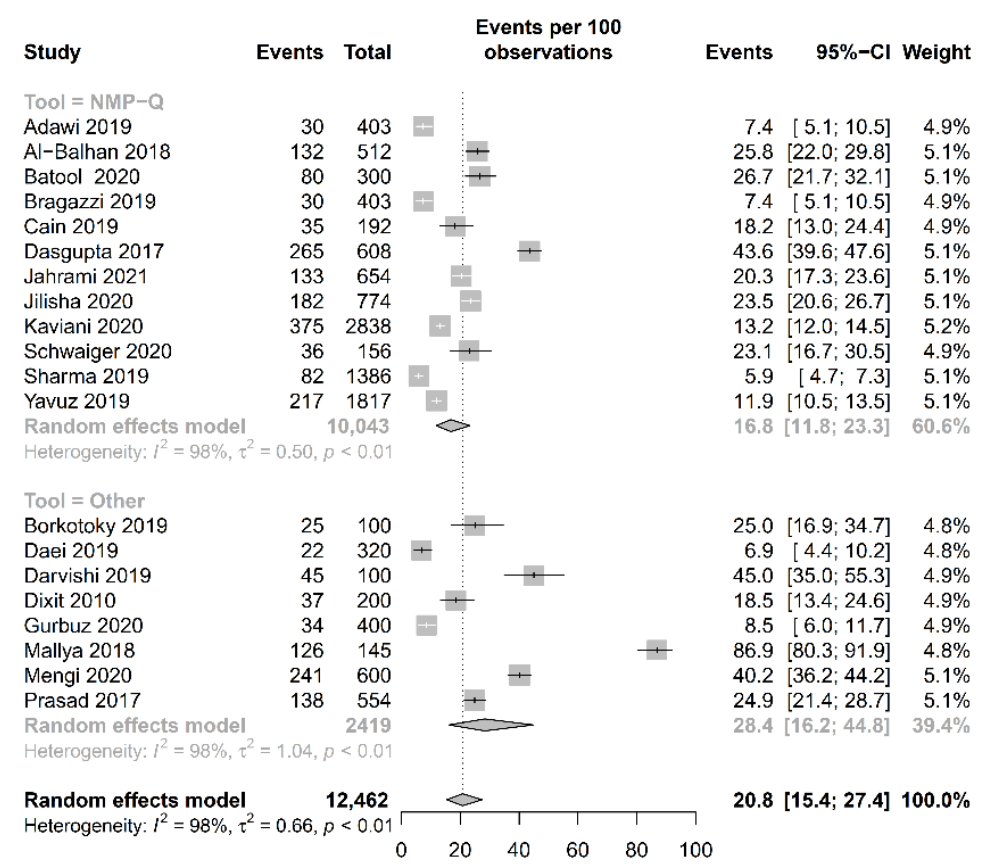

Figure 5. Meta-analysis of the prevalence of severe nomophobia (all populations, by measures).

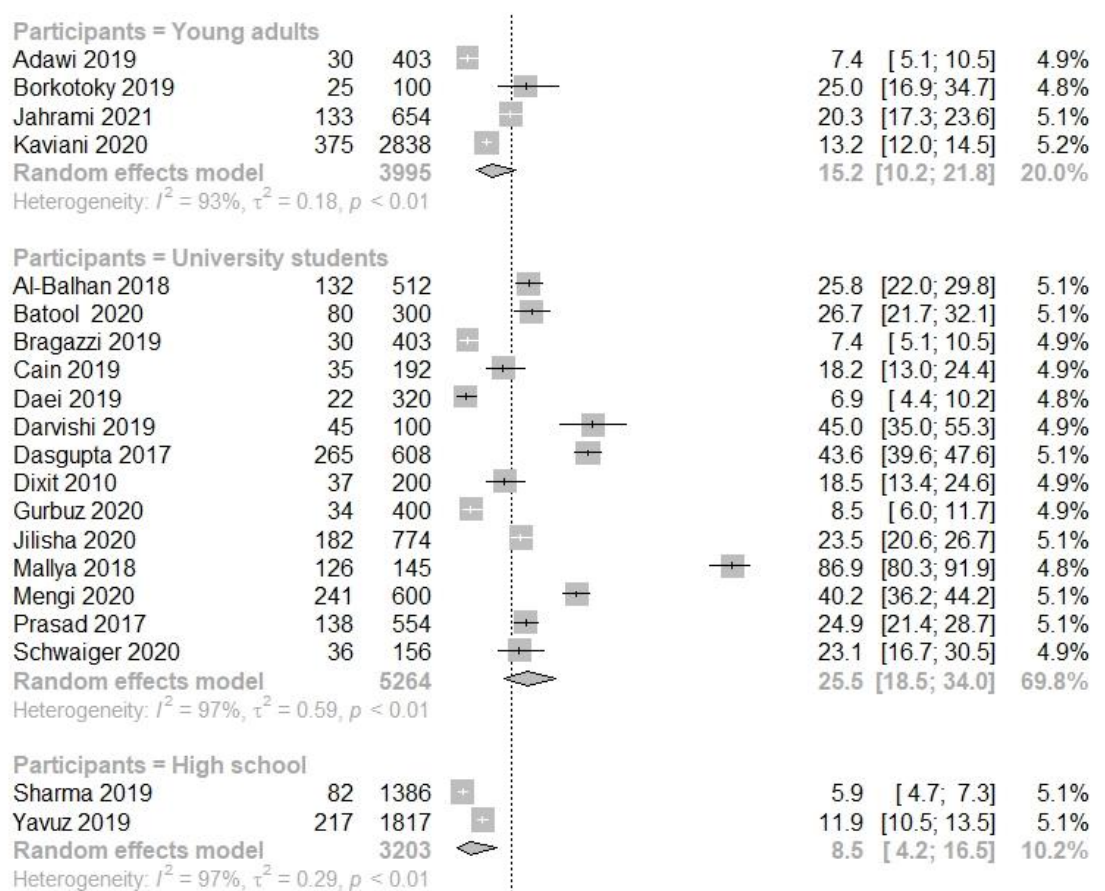

Figure 6. Meta-analysis of the prevalence of severe nomophobia (by populations, all measures).

\section{Discussion}

The purpose of this systematic review was to look at how scientific literature assessed and interpreted nomophobia prevalence. Nomophobia is an emerging issue, and there is no published systematic review or meta-analysis on the prevalence of nomophobia. This meta-analysis is the first to report the global pooled prevalence of nomophobia among several populations using different measurement tools.

The core focus of this systematic review and meta-analysis is on severe nomophobia since it is associated with a serious impact on health because it promotes the development of mental disorders, personality disorders [10,31], and increases the risk of developing 
depression, anxiety, anger, aggressiveness [22], stress, nervousness [32], and sleep disorders $[8,33]$.

The prevalence of moderate to severe nomophobia in all populations using all research tools is $70.76 \%$ [ $95 \% \mathrm{CI} 62.62 \% ; 77.75 \%]$, and the prevalence of severe nomophobia in all populations using all measures is $20.81 \%$ [ $95 \%$ CI $15.45 \%$; $27.43 \%]$. Notably, this finding is similar to the lifetime pooled prevalence of anxiety disorders, which is estimated to be $16.6 \%$ [95\% CI 12.7\%, 21.1\%] [34]. The overlapping prevalence rates between anxiety disorder and nomophobia point to the potential bidirectional relationships between nomophobia and anxiety disorder, suggesting the importance of considering another psychiatric comorbidity when evaluating nomophobia and vice versa $[8,10,29]$.

A recent systematic review showed that nomophobia is more common among women and younger people [35]. The discrepancy in reporting the prevalence of nomophobia is considerable, as the percentages of "at-risk" individuals range from 13 to $79 \%$, and those suffering from it range from 6 to $73 \%$ [35]. Similar to our findings within nomophobia, moderate instances are higher than severe instances [35]. Finally, although methodological differences make it difficult to draw conclusive findings, females and young individuals appear to be more prone to nomophobia [35].

The NMP-Q was used in 12 studies $(60 \%)$, while the rest of the studies opted for the use of other measurement tools, which mainly consisted of researcher-developed measurement tools. The fact that the majority of the studies included in this systematic review and meta-analysis have used the same well-established measurement tool, which is the NMP-Q, has increased the quality and simultaneously decreased the bias of the study. The pooled prevalence of severe nomophobia, using the NMP-Q tool, was estimated to be $16.80 \%$ [ $95 \%$ CI $11.835 ; 23.30 \%$ ], which is similar to the global pooled prevalence of severe nomophobia in all populations using all measures (20.81\% [95\% CI 15.45\%; 27.43\%]). Researcher-developed research tools appeared to be less sensitive in detecting severe nomophobia, compared to the well-established NMP-Q tool, with a prevalence rate of was $28.36 \%$ [95\% CI 16.16; $44.84 \%]$.

Most of the studies that were included in the analysis were conducted in India and the Middle East. On the contrary, the lack of studies reported from other regions, such as Eastern Europe and China, has limited the reliability of the global pooled prevalence obtained from this study. More studies need to be done in these regions to provide an accurate estimation of the global prevalence of nomophobia, have a generalized understanding of the etiology and impact of nomophobia concerning the different cultural norms, and provide a better public health strategy for prevention and screening accordingly.

In this systematic review with meta-analysis, we found that 14 studies $(70 \%)$ were done on university students as they constitute a major sub-group of the anxiety disorders population. Another limitation of this systematic review and meta-analysis is that most populations in the studies were restricted to a narrow age range. Hence, we recommend that future studies should be done on a wider age range, including old adults and the elderly, to have a better estimation of the distribution of nomophobia and it is implications among different age groups.

An intriguing aspect that shall be emphasized is the meta-regressions of severe nomophobia, which showed that age and sex were not a successful predictor of severe nomophobia, and this can be attributed to the narrow age range of the studied population.

The importance of this systematic review is to provide a better understanding of the global prevalence of this modern emerging condition and to focus on the main implication, which is the anticipated increment of rates of nomophobia in the upcoming years, as the world is moving towards a more digital lifestyle. Therefore, efforts for minimizing the implication of this condition are imperative.

The importance of this systematic review is to provide a better understanding of the global prevalence of this modern emerging condition and to focus on the main implication, which is the anticipated increment of rates of nomophobia in the upcoming years, as the world is moving towards a more digital lifestyle and as a result of the current pandemic and 
social isolation, as well as its impact of this pandemic on human behavior and coping and adaptive mechanisms. Assuming that the rate and prevalence of nomophobia would rise in the coming decade, with our assumption that the prevalence of nomophobia will rise over the next decade, precise data on the current prevalence of nomophobia will be critical for future researchers and healthcare providers to compare and contrast the upcoming changes in the trend of this emerging condition, study this phenomenon in terms of causality and effect, and find a method of prevention and treatment to help minimize the implication of this condition.

\section{Conclusions}

The prevalence of severe nomophobia is approximately $21 \%$ in the general adult population. University students appear to be the most impacted by the disorder. Future studies that target a wide range of ages and wider geographical distribution are warranted, to provide a better public health strategy for prevention and screening accordingly.

Author Contributions: Conceptualization, H.J. and N.L.B.; methodology, H.J.; software, H.J.; validation, A.H., N.A., A.A., H.A. and M.A.; formal analysis, H.J.; investigation, A.H., N.A., A.A., H.A. and M.A.; resources, A.H., N.A., A.A., H.A. and M.A.; data curation, A.H., N.A., A.A., H.A. and M.A.; writing—original draft preparation, H.J., A.H., N.A., A.A., H.A. and M.A.; writing-review and editing, N.L.B. and M.H.; visualization, H.J.; supervision, H.J. and A.H. All authors have read and agreed to the published version of the manuscript.

Funding: This research received no external funding.

Institutional Review Board Statement: Not applicable.

Informed Consent Statement: Not applicable.

Conflicts of Interest: The authors declare no conflict of interest.

\section{References}

1. Yavuz, M.; Altan, B.; Bayrak, B.; Gündüz, M.; Bolat, N. The relationships between nomophobia, alexithymia and metacognitive problems in an adolescent population. Turk. J. Pediatr. 2019, 61, 345-351. [CrossRef]

2. Dixit, S.; Shukla, H.; Bhagwat, A.; Bindal, A.; Goyal, A.; Zaidi, A.K.; Shrivastava, A. A study to evaluate mobile phone dependence among students of a medical college and associated hospital of central India. Indian J. Community Med. 2010, 35, 339-341. [CrossRef] [PubMed]

3. Sharma, M.; Amandeep; Mathur, D.M.; Jeenger, J. Nomophobia and its relationship with depression, anxiety, and quality of life in adolescents. Ind. Psychiatry J. 2019, 28, 231-236. [CrossRef] [PubMed]

4. Bahl, R.R.; DeIuliis, D. Nomophobia. In Substance Abuse and Addiction: Breakthroughs in Research and Practice; IGI Global: Hershey, PA, USA, 2019; pp. 295-306.

5. Ozdemir, B.; Cakir, O.; Hussain, I. Prevalence of Nomophobia among University Students: A Comparative Study of Pakistani and Turkish Undergraduate Students. Eurasia J. Math. Sci. Technol. Educ. 2018, 14, 1519-1532. [CrossRef]

6. Kaur, A.; Sharma, P. A descriptive study to assess the risk of developing nomophobia among students of selected nursing colleges Ludhiana, Punjab. Int. J. Psychiatr. Nurs. 2015, 1, 1-6. [CrossRef]

7. Bhattacharya, S.; Bashar, M.A.; Srivastava, A.; Singh, A. NOMOPHOBIA: NO MObile PHone PhoBIA. J. Fam. Med. Prim. Care 2019, 8, 1297-1300. [CrossRef] [PubMed]

8. Jahrami, H.; Abdelaziz, A.; Binsanad, L.; Alhaj, O.A.; Buheji, M.; Bragazzi, N.L.; Saif, Z.; BaHammam, A.S.; Vitiello, M.V. The Association between Symptoms of Nomophobia, Insomnia and Food Addiction among Young Adults: Findings of an Exploratory Cross-Sectional Survey. Int. J. Environ. Res. Public Health 2021, 18, 711. [CrossRef] [PubMed]

9. Yildirim, C.; Correia, A.-P. Exploring the dimensions of nomophobia: Development and validation of a self-reported questionnaire. Comput. Hum. Behav. 2015, 49, 130-137. [CrossRef]

10. Bragazzi, N.L.; Del Puente, G. A proposal for including nomophobia in the new DSM-V. Psychol. Res. Behav. Manag. 2014, 7, 155-160. [CrossRef]

11. Liberati, A.; Altman, D.G.; Tetzlaff, J.; Mulrow, C.; Gøtzsche, P.C.; Ioannidis, J.P.A.; Clarke, M.; Devereaux, P.J.; Kleijnen, J.; Moher, D. The PRISMA statement for reporting systematic reviews and meta-analyses of studies that evaluate healthcare interventions: Explanation and elaboration. BMJ 2009, 339, b2700. [CrossRef]

12. Lin, L.; Chu, H. Quantifying publication bias in meta-analysis. Biometrics 2018, 74, 785-794. [CrossRef]

13. R. 4.0.3. Available online: https://www.R-project.org/ (accessed on 5 May 2020).

14. Viechtbauer, W. Conducting meta-analyses in R with the metafor package. J. Stat. Softw. 2010, 36, 1-48. [CrossRef] 
15. Mallya, N.V.; DR, S.K.; Mashal, S. A study to evaluate the behavioral dimensions of "Nomophobia" and attitude toward smartphone usage among medical students in Bengaluru. Natl. J. Physiol. Pharm. Pharmacol. 2018, 8, 1553-1557. [CrossRef]

16. Mengi, A.; Singh, A.; Gupta, V. An institution-based study to assess the prevalence of Nomophobia and its related impact among medical students in Southern Haryana, India. J. Fam. Med. Prim. Care 2020, 9, 2303-2308. [CrossRef]

17. Jilisha, G.; Venkatachalam, J.; Menon, V.; Olickal, J.J. Nomophobia: A Mixed-Methods Study on Prevalence, Associated Factors, and Perception among College Students in Puducherry, India. Indian J. Psychol. Med. 2019, 41, 541-548. [CrossRef] [PubMed]

18. Borkotoky, C.; Saikia, J. A study on prevalence of mobile phone dependence among youth of Jorhat district. Indian J. Health Wellbeing 2019, 10, 246-248.

19. Dasgupta, P.; Bhattacherjee, S.; Dasgupta, S.; Roy, J.K.; Mukherjee, A.; Biswas, R. Nomophobic behaviors among smartphone using medical and engineering students in two colleges of West Bengal. Indian J. Public Health 2017, 61, 199-204. [CrossRef]

20. Prasad, M.; Patthi, B.; Singla, A.; Gupta, R.; Saha, S.; Kumar, J.K.; Malhi, R.; Pandita, V. Nomophobia: A Cross-sectional Study to Assess Mobile Phone Usage Among Dental Students. J. Clin. Diagn. Res 2017, 11, zc34-zc39. [CrossRef] [PubMed]

21. Daei, A.; Ashrafi-Rizi, H.; Soleymani, M.R. Nomophobia and Health Hazards: Smartphone Use and Addiction Among University Students. Int. J. Prev. Med. 2019, 10, 202. [CrossRef]

22. Darvishi, M.; Noori, M.; Nazer, M.R.; Sheikholeslami, S.; Karimi, E. Investigating Different Dimensions of Nomophobia among Medical Students: A Cross-Sectional Study. Open Access Maced. J. Med. Sci. 2019, 7, 573-578. [CrossRef]

23. Gurbuz, I.B.; Ozkan, G. What is Your Level of Nomophobia? An Investigation of Prevalence and Level of Nomophobia Among Young People in Turkey. Community Ment. Health J. 2020, 56, 814-822. [CrossRef]

24. Batool, I.; Zahid, A. Nomophobia an Emerging Fear: An Experimental Exploration among University Students. Peshawar J. Psychol. Behav. Sci. (PJPBS) 2019, 5, 67. [CrossRef]

25. Schwaiger, E.; Tahir, R. Nomophobia and its predictors in undergraduate students of Lahore, Pakistan. Heliyon 2020, 6, e04837. [CrossRef]

26. Kaviani, F.; Robards, B.; Young, K.L.; Koppel, S. Nomophobia: Is the Fear of Being without a Smartphone Associated with Problematic Use? Int. J. Environ. Res. Public Health 2020, 17, 6024. [CrossRef] [PubMed]

27. Adawi, M.; Zerbetto, R.; Re, T.S.; Bisharat, B.; Mahamid, M.; Amital, H.; Del Puente, G.; Bragazzi, N.L. Psychometric properties of the Brief Symptom Inventory in nomophobic subjects: Insights from preliminary confirmatory factor, exploratory factor, and clustering analyses in a sample of healthy Italian volunteers. Psychol. Res. Behav. Manag. 2019, 12, 145-154. [CrossRef] [PubMed]

28. Bragazzi, N.L.; Re, T.S.; Zerbetto, R. The Relationship Between Nomophobia and Maladaptive Coping Styles in a Sample of Italian Young Adults: Insights and Implications From a Cross-Sectional Study. JMIR Ment. Health 2019, 6, e13154. [CrossRef]

29. Al-Balhan, E.M.; Khabbache, H.; Watfa, A.; Re, T.S.; Zerbetto, R.; Bragazzi, N.L. Psychometric evaluation of the Arabic version of the nomophobia questionnaire: Confirmatory and exploratory factor analysis-Implications from a pilot study in Kuwait among university students. Psychol. Res. Behav. Manag. 2018, 11, 471-482. [CrossRef]

30. Cain, J.; Malcom, D.R. An Assessment of Pharmacy Students' Psychological Attachment to Smartphones at Two Colleges of Pharmacy. Am. J. Pharm. Educ. 2019, 83, 7136. [CrossRef]

31. Lee, S.; Kim, M.; Mendoza, J.S.; McDonough, I.M. Addicted to cellphones: Exploring the psychometric properties between the nomophobia questionnaire and obsessiveness in college students. Heliyon 2018, 4, e00895. [CrossRef] [PubMed]

32. Gutiérrez-Puertas, L.; Márquez-Hernández, V.V.; Aguilera-Manrique, G. Adaptation and Validation of the Spanish Version of the Nomophobia Questionnaire in Nursing Studies. Comput. Inf. Nurs. 2016, 34, 470-475. [CrossRef] [PubMed]

33. Gentina, E.; Tang, T.L.-P.; Dancoine, P.-F. Does Gen Z's emotional intelligence promote iCheating (cheating with iPhone) yet curb iCheating through reduced nomophobia? Comput. Educ. 2018, 126, 231-247. [CrossRef]

34. Remes, O.; Brayne, C.; van der Linde, R.; Lafortune, L. A systematic review of reviews on the prevalence of anxiety disorders in adult populations. Brain Behav. 2016, 6, e00497. [CrossRef] [PubMed]

35. León-Mejía, A.C.; Gutiérrez-Ortega, M.; Serrano-Pintado, I.; González-Cabrera, J. A systematic review on nomophobia prevalence: Surfacing results and standard guidelines for future research. PLoS ONE 2021, 16, e0250509. [CrossRef] [PubMed] 